\title{
Correlatos valorativos do crescimento pós-traumático em uma amostra brasileira $^{1}$
}

\author{
Value correlates of posttraumatic growth in an brazilian sample
}

\author{
Emerson Diógenes de Medeiros ${ }^{2}$ \\ Ricardo Neves Couto ${ }^{3}$ \\ Patrícia Nunes da Fonseca 4 \\ Rislay Carolinne Silva Brito ${ }^{5}$ \\ Laís Serra Castro ${ }^{6}$
}

\begin{abstract}
RESUMO: Este estudo objetivou verificar a relação existente entre o Crescimento Pós-Traumático e os valores humanos, tendo como base a Teoria Funcionalista. Para tanto, participaram 212 pessoas com idades variando de 18 a 86 anos $(m=28$ anos e $d p=11,45)$, a maioria mulheres $(55,4 \%)$, solteiros $(67,2 \%)$ e com ensino superior incompleto $(37,6 \%)$. Os participantes responderam ao Posttrumatic Growth Inventory (PTGI) e o Questionário de Valores Humanos Básicos. Foi realizada análise de correlação ( $r$ de Pearson) e regressões lineares múltiplas. Os resultados indicaram relações entre a subfunção existência com todos os fatores do PTGI, além das subfunções interativa, normativa, existência e realização apresentarem relação preditiva com o fator geral. Conclui-se que os resultados foram congruentes com a literatura especializada. Contribuindo para o avanço cientifico da temática, propõe-se uma investigação inovadora no trabalho da adaptação frente ao trauma e reafirma a centralidade dos valores na Psicologia Social.
\end{abstract}

Palavras-chave: psicologia positiva; psicologia social; crescimento pós-traumático; valores humanos.

\begin{abstract}
This study aimed to verify the existing relationship between the Post-traumatic Growth and the human values, based on the Fundamentalist Theory. To this end, 212 people attended with ages ranging $18-86$ years old (mean $=28$ years, SD $=11,45$ ) in most women (55.4\%), single (67.2\%) and incomplete higher education (37.6\%), which responded to Posttrumatic Growth Inventory (PTGI) and the Questionnaire of Basic Human Values. Correlation analysis was performed (Pearson's $r$ ) and multiple linear regressions. Resulting relationship between the subfunction existence with all PTGI factors, and to subfunction interactive, normative, existence and promotion presents relationship with the general factor. We conclude that the results were consistent with the literature. In order to contribute to the scientific advance of this topic, it is proposed an innovative investigation in the adaptation toward the trauma, as well as restate the centrality of the values in the Social Psychology.
\end{abstract}

Keywords: positive psychology; social psychology; post-traumatic growth; human values.

\footnotetext{
${ }^{1} \mathrm{O}$ trabalho que deu origem ao manuscrito contou com apoio do CNPq e FAPEPI por meio de financiamento de projetos de pesquisas do primeiro (CNPq e FAPEPI) e terceiro (CNPq) autores, além de bolsa de Mestrado (CNPq) ao segundo. Aproveitamos para demonstrar nossa gratidão a estas instituições.

2 Doutor em Psicologia Social pelo Departamento de Psicologia da Universidade Federal da Paraíba. Professor Adjunto do Departamento de Psicologia da Universidade Federal do Piauí. Coordenador do Laboratório de Avaliação Psicológica do Delta - LABAP - Parnaíba, PI, Brasil. E-mail: emersondiogenes@mail.com.

${ }^{3}$ Graduado em Psicologia pela Universidade Federal do Piauí. Mestrando em Psicologia Social pelo Departamento de Psicologia da Universidade Federal da Paraíba - João Pessoa, PB, Brasil.

${ }^{4}$ Doutora em Psicologia Social pelo Departamento de Psicologia da Universidade Federal da Paraíba. Professora Adjunta do Departamento de Psicopedagogia da Universidade Federal da Paraíba - João Pessoa, PB, Brasil.

${ }^{5}$ Graduanda em Psicologia pelo Departamento de Psicologia da Universidade Federal do Piauí - Parnaíba, PI, Brasil.

${ }^{6}$ Graduanda em Psicologia pelo Departamento de Psicologia da Universidade Federal do Piauí - Parnaíba, PI, Brasil.
} 


\section{Introdução}

Neste estudo, objetiva-se conhecer em que medida os valores humanos explicam a ocorrência do Crescimento Pós-Traumático (CPT), especificamente verificar a relação entre as subfunções valorativas e as dimensões do Posttraumatic Growth Inventory (PTGI) e apresentar dados acerca de vivências traumáticas. A utilização dos valores humanos como preditores de diversos fenômenos apresenta-se em muitos estudos. Entretanto, não se encontrou na literatura pesquisas que abordassem a sua relação com o CPT.

Acontecimentos adversos, incontroláveis e imprevisíveis, denominados como crises ou eventos estressores estão presentes na vida de todo ser humano. Caracteriza-se um trauma quando a resposta é dada por um medo intenso ou uma sensação de impotência. Entretanto, a forma como cada pessoa encara a vivência destes eventos é subjetiva, destacados como potencialmente traumáticos por uma situação não gerar nenhuma consequência negativa para uma pessoa e para outra, desencadear perturbações físicas e psicológicas (Madeira, 2013). Não obstante, caracterizando o CPT, eles se tornam oportunidades para crescer através da dor e para abrir novos horizontes, assumindo uma nova perspectiva na vida e desenvolvendo a perseverança através das dificuldades (Lau et al., 2013).

No tocante a vivência de um evento traumático, Cann et al. (2010) asseveram que pode levar o indivíduo a reexaminar os elementos principais das crenças que definem a sua visão de mundo. Quanto maior a necessidade de reexaminar as crenças, maior a probabilidade de experienciar crescimento após o trauma (Triplett, Tedeschi, Cann, Calhoun, \& Reeve, 2012).

Neste cenário de controvérsias quanto a reações frente ao trauma, a concepção de que o ser humano é capaz de transformar experiências adversas em aprendizagens, tem sido um tema muito tratado na Literatura, Poesia e Filosofia. Já a Psicologia, durante muitos anos, ignorou esta perspectiva, enfatizando apenas o alívio de sintomas e correções dos danos (Reppold, Gurgel, \& Schiavon, 2015).

Entretanto, a partir do foco da Psicologia Positiva e de sua expansão desde o início da década de 90, têm-se buscado um equilíbrio dos estudos, investigando também o que há de melhor no indivíduo (Vázquez, 2013). Dessa forma, essa perspectiva questiona o foco da psicologia tradicional que se voltava unicamente para o desenvolvimento de uma patologia após o indivíduo passar por um evento estressor, por exemplo, o transtorno do estresse póstraumático. Portanto, ela apresenta estudos, entre outros com respostas positivas frente ao trauma, com o CPT.

Os estudos sobre CPT, mesmo se mostrando de forma crescente, ainda não demonstram conclusões sobre sua ocorrência. Logo, faz-se necessário preencher lacunas acerca dos antecedentes e das variáveis preditivas deste fenômeno. O qual recebe essa denominação devido ao nível atingido pela pessoa após a vivência traumática, haja vista que o seu funcionamento após o trauma supera a situação anterior, proporcionando um reconhecimento de suas forças e virtudes (Fonseca, 2011). De acordo com Osei-Bonsu, Weaver, Eisen e Wal (2012) existem evidências dos benefícios percebidos após experiências de vida desafiadoras que são documentadas desde os anos 1980. Segundo Calhoun e Tedeschi (2012), no início dos anos de 1960, já eram reconhecidas as mudanças psicológicas positivas como possibilidade de ocorrência em situações de alto stress. 
Tedeschi e Calhoun (1996) estudaram e organizaram as mudanças psicológicas positivas experimentadas pelo indivíduo como resultado de um processo de luta frente à vivência de grandes crises ou eventos traumáticos, conceituando, a partir disto, o CPT em três categorias: mudança de significado do self ou auto percepção, mudança de significado das relações com os outros e mudanças na filosofia de vida, a seguir detalhadas.

A primeira categoria diz respeito à pessoa, perceber-se mais forte e confiante em si mesma, com mais experiência, com capacidade de enfrentar dificuldades futuras e uma maior percepção de força individual. Além de estar relacionada ao reconhecimento de mais recursos. Outra mudança categorizada aparece nas relações interpessoais. É notório mais conforto e confiança ao revelar seus pensamentos e emoções aos outros, além da sensação de maior compaixão por pessoas que também passam por dificuldades (Fonseca, 2011; Calhoun \& Tedeschi, 2012). A terceira categoria é característica de pessoas que mudam de prioridades e opções em vários domínios de suas vidas, a saber: espiritual, religioso e existencial. Originalmente, estes domínios foram organizados para definir o construto crescimento pós-traumático.

Contudo, segundo investigações recentes (Duarte, 2012; Taku, Kilmer, Cann, Tedeschi, \& Calhoun, 2012), influenciadas por estudos oriundos da Psicologia Positiva, tais domínios foram reformulados em cinco: (1) aumento da apreciação de vida no geral; (2) relações interpessoais mais significativas; (3) aumento do sentimento de força pessoal; (4) mudanças de prioridades; e (5) uma vida espiritual e existencial mais rica.

Logo, o CPT é caracterizado como um construto multidimensional, no qual os indivíduos podem experimentar mudanças positivas em alguns destes domínios e negativas em outros (Ramos \& Leal, 2013). No entanto, os resultados acerca da melhor representação estrutural do construto são ambíguos, pois, segundo estudiosos sobre o tema, como Thege, Kovács \& Balog (2013), não fica claro se o crescimento pós-traumático é melhor representado por um ou mais fatores. Tenha-se em conta que, nesta oportunidade, será considerado o CPT representado por cinco fatores.

\section{Variáveis relacionadas ao CPT}

Encontram-se estudos que associam variáveis sóciodemográficas com o crescimento percebido. Quanto ao sexo, Ramos e Leal (2013), mesmo não generalizando os resultados, sugerem que as mulheres experimentam mais crescimento do que os homens. Tedeschi e Calhoun (2004) afirmam que pessoas com níveis de escolaridade mais elevados são geralmente mais propensos a apresentar mais crescimentos ou benefícios. Em relação à idade, é provável que os mais jovens possam reportar mais crescimento, visto que os seus esquemas cognitivos sobre si e sobre o mundo ainda não estão consolidados e, como tal, são mais suscetíveis a rupturas e mudanças (Fonseca, 2011; Vázquez, 2013).

No tocante às relações encontradas com os cinco traços básicos de personalidade, Calhoun, Tedeschi, Cann e Hanks (2010) apontam que a extroversão e a abertura à experiência têm maior relação com o CPT. Segundo estes autores, é possível que pessoas com estas características apresentem mais emoções e cognições positivas em situações de adversidade. Elas têm, portanto, uma melhor base para pôr em prática os mecanismos de reflexão e reconstrução cognitiva pós-traumática. 
A ruminação, caracterizada como uma cadeia de pensamentos repetitivos (Zanon, Borsa, Bandeira, \& Hutz, 2012), está presente na adaptação frente ao trauma (Tedeschi, 2011). Concomitante a isto, Vázquez, Castilla e Hervás (2008) destacam o apoio social como uma oportunidade de fala e escuta de pessoas que estiveram em uma situação semelhante, uma ajuda recíproca que auxilia no processo de crescimento.

Outros fatores relevantes na previsão do crescimento pós-traumático são as crenças religiosas e espirituais. Segundo Bert (2011), estas podem se tornar a base de sua filosofia de vida, proporcionando forças, orientações e assistência no esforço investido na compreensão dos eventos traumáticos. Subandi, Achmad, Kurniati e Febri (2014) encontraram um papel significativo da espiritualidade na explicação do crescimento. Gall, Kristjansson, Charbonneau e Florack (2009) concluíram que a espiritualidade e religiosidade dão uma orientação individual sobre como se comportar e avaliar uma situação adversa, que medidas devem ser tomadas, bem como decidir qual a estratégia de enfrentamento será usada.

Há um recente interesse na influência da gratidão, posto que indivíduos que são gratos, após a vivência de um trauma, formam cognições positivas que ajudam restabelecer o significado do mundo (Wu, Zhou, Liu, \& Chen, 2014). Vivenciam emoções positivas que se alarguem o repertorio de ações, pensamentos e otimismo durante o tempo de adaptação (Fredrickson, 2004). Os resultados de um estudo longitudinal com adolescentes chineses sobreviventes de terremoto sugerem que a gratidão é um fator preditivo estável para o desenvolvimento do crescimento pós-traumático (Zhou \& Wu, 2015).

Tais variáveis aqui apresentadas estão teoricamente relacionadas aos valores humanos. Por exemplo, espiritualidade está relacionada à subfunção normativa, suporte social à subfunção interativa. Portanto, a investigação sobre quais características determinam a ocorrência do fenômeno seria enriquecida a partir de uma visão direcionada às prioridades valorativas dos indivíduos e nesta oportunidade, buscar-se-á o padrão de associação das dimensões do CPT com os valores humanos, construto de cunho social, apreendido no processo de socialização.

\section{Valores humanos}

Rokeach (1973) define valores como crenças hierárquicas baseadas nas necessidades individuais e que indicam o que é adequado ou não realizar em uma determinada situação. Segundo Ros (2006), considerar os seus antecedentes históricos é quase que arbitrário, o que se encontra são autores que podem ser mencionados como representantes de duas tradições distintas. Uma delas trata-se da tradição sociológica, com autores como Hofstede, e seus estudos transculturais dos valores, e Inglehart que defende as mudanças na ordem dos valores de acordo com a ordem econômica. A outra tradição apresenta-se como psicológica, com autores como Maslow, Rokeach e Schwartz, o qual sugere uma tipologia universal das motivações subjacentes aos valores das pessoas.

Neste estudo será abordado como âncora uma nova perspectiva teórica proposta por Gouveia $(1998,2003)$ : Teoria Funcionalista. Representando a tradição psicológica, Gouveia parte de críticas e lacunas identificadas para apresentar sua teoria, a qual é encarada como mais parcimoniosa e integradora, além de não visar substituir os modelos anteriores. Seu foco principal compreende as funções valorativas, as quais entendem os valores como aspectos psicológicos que guiam os comportamentos e representam cognitivamente as 
necessidades humanas. São, portanto, princípios-guia desejáveis, que têm o papel de assegurar a sobrevivência, manutenção e evolução da sociedade, explicando atitudes e comportamentos de seus membros (Gouveia, 2013).

Segundo esta teoria, os valores apresentam duas dimensões principais: o eixo horizontal corresponde ao tipo de orientação e o vertical ao tipo de motivador (Gouveia, 2013). O cruzamento destes eixos (social, central e pessoal / materialista e humanitário) gera seis subfunções, distribuídas entre os critérios de orientação social (interativa e normativa), central (suprapessoal e existência) e pessoal (experimentação e realização), e os tipos de motivadores: idealista (interativa, suprapessoal e experimentação) e materialista (normativa, existência e realização).

No tocante a organização funcional dos valores, a descrição das seis subfunções leva em consideração que os valores centrais constituem a principal fonte em que são ancorados os outros valores. A partir disso, a seguir, os valores serão apresentados, considerando as duas funções: tipo de orientação e o tipo motivador, descrevendo os conteúdos específicos de cada subfunção (Gouveia, 2013).

Subfunção existência: possui um tipo motivador materialista e tipo orientador central. Tendo como propósito principal assegurar as condições básicas para a sobrevivência biológica e psicológica do indivíduo é constituída pelos valores: saúde, sobrevivência e estabilidade pessoal. O primeiro representa a busca de um grau adequado de saúde e a abstenção de eventos ameaçadores. O valor sobrevivência geralmente é o princípio guia de pessoas socializadas em contextos de escassez ou que não possuem os recursos econômicos básicos. Por fim, pessoas que primam pelo valor estabilidade pessoal buscam ter uma vida organizada e planejada, garantindo sua própria existência.

Subfunção realização: representa o motivador materialista e têm uma orientação pessoal. Pessoas orientadas por esta subfunção focam em realizações materiais, pessoais e imediatas, voltadas para o aqui e agora. Sendo esta constituída pelos valores: êxito, enfatizando a eficiência e o alcance de metas, prestígio, pessoas tendem a procurar ter uma imagem pública reconhecida e poder, ênfase dada ao princípio da hierarquia, objetivando ser o chefe.

Subfunção normativa: com tipo motivador materialista, mas com orientação social, esta subfunção representa a necessidade de controle e as pré-condições imprescindíveis para alcançar todas as necessidades. Representada pelos valores: obediência, religiosidade e tradição. O primeiro evidencia a importância de obedecer e cumprir deveres e obrigações diárias, respeito pelos pais e mais velhos. Quanto ao valor religiosidade, este não depende de qualquer preceito religioso, existindo o reconhecimento de uma entidade superior em que se busca certeza e harmonia social para uma vida social pacífica e segura. O valor tradição indica respeito aos padrões morais seculares além de contribuição para a harmonia social.

Subfunção Suprapessoal: com orientação central e um tipo motivador humanitário, representa as necessidades de estética, cognição e autorrealização. Fazem parte desta subfunção os seguintes valores: conhecimento, maturidade e beleza. Quanto ao conhecimento, pessoas que priorizam este valor buscam conhecimentos novos e atuais, além de informações sobre assuntos gerais. $O$ valor maturidade representa a necessidade de autorrealização. Beleza representa claramente a preocupação com a estética e, independente da natureza do objeto (material ou imaterial), as pessoas cultivam o belo. 
Subfunção Experimentação: apresenta um motivador idealista com orientação pessoal. Seus valores representam a necessidade fisiológica de satisfação, em sentido amplo, ou o princípio do prazer (hedonismo). Fazem parte desta subfunção tais valores descritos: emoção, representa a necessidade fisiológica de excitabilidade e busca de experiências perigosas, arriscadas; prazer, corresponde a uma necessidade orgânica de satisfação em sentido mais amplo com uma fonte de satisfação inespecífica e sexualidade, denota a necessidade do sexo, tem sido tratado como indicador ou fator de moralidade.

Subfunção interativa: representa o tipo motivador idealista com orientação social, sendo características de pessoas mais jovens e orientadas para relações íntimas estáveis. Tem como valores que a caracterizam: afetividade, relacionado com aspectos da vida social, enfatizando relacionamentos íntimos; apoio social, expressa a segurança no sentido de não se sentir sozinho no mundo e, quando necessitar, a obtenção de ajuda; e convivência, indica a ideia de pertença a um grupo social e não viver sozinho.

Um estudo realizado por Medeiros (2011), com aproximadamente 50.000 pessoas, abrangendo as cinco regiões do Brasil e mais onze países, apresenta evidências da adequação da teoria funcionalista dos valores humanos. Ademais, essa teoria é utilizada em estudos que abordam os valores como preditores de diversos fenômenos psicossociais, evidenciando a importância desse construto na Psicologia Social.

\section{Método}

\section{Amostra}

Utilizou-se, como critério de inclusão na amostra, ter sofrido ou passado pelo menos por um dos acontecimentos estressantes ou traumáticos indicados na Lista de acontecimentos adversos (Maia \& Resende, 2008), sendo a amostra final, por conveniência, composta por 212 pessoas de cidades do nordeste brasileiro, na maioria oriundos de Parnaíba (53,04\%), Ilha Grande do Piauí (27,02\%), com idades variando entre 18 a 86 anos $(M=27,9, D P=11,44)$, sendo a maioria do sexo feminino $(53,8 \%)$ e solteira $(65,2 \%)$. Deste total, $36,7 \%$ das pessoas declararam possuir ensino superior incompleto como grau de escolaridade. Comparando-se com outras pessoas que residiam em suas cidades, a maioria declarou fazer parte da classe média $(56,5 \%)$ e com renda familiar média aproximada de $\mathrm{R} \$$ $2.184,85(d p=2.364,04)$.

\section{Instrumentos}

Lista de Acontecimentos Adversos. Este instrumento foi traduzido para o português por Maia e Resende (2008) a partir da Life Stressor Checklist-Revised (L.S.C.-R.) de Wolfe, Kimerling, Brown, Chresman e Levin (1996). Consiste numa lista de 30 acontecimentos de vida adversos. Nele, os participantes marcam quais dos eventos que teriam acontecido em suas vidas foram considerados como traumáticos, ou ainda, têm a possibilidade de apontarem outros que não constavam na lista.

Posttraumatic Growth Inventory (PTGI). Esse instrumento foi adaptado e validado para o contexto brasileiro por Medeiros, Couto, Fonseca, Silva, \& Medeiros (No prelo). Originalmente desenvolvido por Tedeschi e Calhoun (1996), avalia o grau de mudanças 
positivas percebidas pelo indivíduo após um acontecimento adverso. Uma escala de 21 itens que possui 5 fatores e apresentou tais índices de consistência interna: Relação com os outros (7 itens; $\alpha=0,86$ ), Novas possibilidades (5 itens; $\alpha=0,77$ ), Força Pessoal (4 itens; $\alpha=0,70$ ), Mudança Espiritual ( 2 itens; $\alpha=0,79$ ) e Apreciação da Vida ( 3 itens; $\alpha=0,70$ ), sendo que o fator geral (somatório de todos os itens) corresponde ao índice de crescimento póstraumático (21 itens; $\alpha=0,92$ ). Os itens, a exemplo do item 2: "Dou mais valor à minha vida" e do item 19: "Descobri que sou mais forte do que pensava", são respondidos com escala de respostas do tipo Likert que varia de 0 (Não experimentei mudança como resultado desse trauma) a 5 (Mudei completamente), em que consideram ter mudado em consequência de um determinado trauma (e.g. item)

Questionário de Valores Básicos (QVB; Gouveia, 2003; Gouveia, Milfont, Fischer, \& Santos, 2008). Compõe-se de dezoito itens (valores específicos) distribuídos nas seis subfunções psicossociais: experimentação (emoção, prazer e sexual), realização (êxito, poder e prestígio), existência (estabilidade pessoal, saúde e sobrevivência), suprapessoal (beleza, conhecimento e maturidade), normativa (obediência, religiosidade e tradição) e interativa (afetividade, apoio social e convivência). Para cada um dos valores existem dois descritores que retratam o sentido dado, por exemplo, o valor afetividade sugere "ter uma relação de afeto profunda e duradora" e "ter alguém para compartilhar seus êxitos e fracassos. 0 participante deve indicar em que medida considera cada valor importante como um princípio-guia em sua vida, utilizando escala de resposta de sete pontos, variando de $1=$ Totalmente não importante a 7 = De extrema importância. As seis subfunções, no contexto nacional, especificamente na região Nordeste, apresentam consistência interna variando de 0,39 (subfunção realização) a 0,55 (subfunção existência), bem como indicadores de ajuste satisfatórios $[G F I=0,95$ e RMSEA (IC90\%) =0,06 (0,06 - 0,06)] (Medeiros, 2011).

Questões sociodemográficas. Conjunto de perguntas que objetivam caracterizar a amostra, a exemplo de questões sobre a idade, o sexo, a renda, nível de escolaridade e o estado civil.

\section{Procedimentos}

Para a aplicação dos instrumentos, dois bolsistas de iniciação científica e dois colaboradores treinados, visitavam locais públicos (praças, parques) e residências em cidades do nordeste brasileiro e convidavam as pessoas para participarem da pesquisa. Neste momento, eram informados o objetivo geral do estudo, o caráter voluntário da participação, bem como o anonimato das respostas e a possibilidade de deixar o estudo a qualquer momento, sem nenhum ônus. Após aceitarem participar do estudo, os participantes eram convidados a assinar o Termo de Consentimento Livre e Esclarecido. Os instrumentos eram respondidos de forma individual, sendo necessários, em média, 20 minutos para sua conclusão.

Foram asseguradas todas as recomendações éticas relacionadas às pesquisas com seres humanos, conforme o disposto na Resolução 466/2012 do Conselho Nacional de Saúde, sendo a realização do estudo aprovado pelo Comitê de Ética em Pesquisa da Universidade Federal do Piauí (Parecer n 886.238/2014). 


\section{Análise de dados}

O software IBM SPSS (em sua versão 21) foi utilizado para calcular as estatísticas descritivas com o fim de caracterização da amostra. Com este mesmo software foram realizados análise de correlações ( $r$ de Pearson) e análises de regressão múltiplas. Tais análises permitem verificar o padrão de associação entre os índices de crescimento e as subfunções valorativas (Tabachnick \& Fidell, 2012).

\section{Resultados}

A amostra foi composta por pessoas que relataram vivência de um evento considerado traumático, verificou-se que $47,02 \%$ dos participantes informaram que vivenciaram mais de um ao longo da vida. Dentre os mencionados, os que apareceram com mais frequências foram: "morte inesperada de um familiar ou amigo próximo (79 vezes), morte de um familiar ou amigo próximo (51 vezes), assistir a violência entre membros de família (27 vezes), assistir a um acidente grave ( 25 vezes), divórcio parental ( 25 vezes), problemas financeiros graves (25 vezes), experiência de abuso emocional (ex. ridicularizado, ignorado) (25 vezes), ser assaltado ou sofrer um ataque físico (23 vezes), além de outros acontecimentos traumáticos que não constavam na lista (27 vezes) e relatar não ter vivenciado algum evento diretamente, mas ter tido conhecimento de ocorrência de acontecimentos com pessoas próximas (24 vezes). Pretendendo conhecer em que medida e direção as subfunções valorativas se relacionam com as dimensões do CPT, ou seja, como os valores explicam as mudanças positivas experimentadas pelos participantes após essas vivências adversas relatadas, foram realizadas análises de correlação e regressões lineares múltiplas. Neste caso, as dimensões equivalem às pontuações nos fatores do PTGI, os quais correspondem à estrutura encontrada e validada do PTGI no estudo para o contexto brasileiro por Medeiros et al. (No prelo).

A Tabela 1 apresenta as correlações de Pearson entre esses cinco fatores, além do fator geral do CPT, com as seis subfunções valorativas. Apesar de existirem outras relações, apenas a subfunção existência apresenta relações positivas e estatisticamente significativas com todas as cinco dimensões: relação com os outros $(r=0,16 ; p<0,05)$; novas possibilidades $(r=0,14 ; p<0,14)$; mudança pessoal $(r=0,14 ; p<0,05)$; mudança espiritual ( $r$ $=0,15 ; p<0,05)$ e apreciação da vida $(r=0,15 ; p<0,05)$.

O fator geral do CPT se correlacionou positivamente com todas as subfunções que apresentam o tipo motivador materialista: normativa $(r=0,24 ; p<0,05)$, existência $(r=0,19$; $p<0,05)$ e realização $(r=0,22 ; p<0,05)$. Entretanto, com as do tipo motivador idealista, esta relação ocorreu apenas com a subfunção interativa $(r=0,20 ; p<0,05)$. 
Tabela 1 - Correlatos Valorativos das dimensões do CTP

\begin{tabular}{|c|c|c|c|c|c|c|}
\hline & \multirow[b]{2}{*}{1} & \multirow[b]{2}{*}{2} & \multicolumn{3}{|c|}{ Subfunções Valorativas } & \multirow[b]{2}{*}{6} \\
\hline & & & 3 & 4 & 5 & \\
\hline Relação com os outros & $0,20 * *$ & $0,24 * *$ & 0,07 & $0,16^{*}$ & $-0,22$ & $0,17^{*}$ \\
\hline Novas possibilidades & 0,12 & 0,13 & $0,14 *$ & $0,14^{*}$ & 0,01 & $0,17^{*}$ \\
\hline Mudança Pessoal & 0,10 & 0,12 & 0,10 & $0,14^{*}$ & 0,04 & $0,22 * *$ \\
\hline Mudança Espiritual & 0,13 & $0,40 * *$ & $-0,02$ & $0,15^{*}$ & $-0,16$ & 0,02 \\
\hline Apreciação da Vida & $0,21 *$ & 0,06 & 0,13 & $0,15^{*}$ & 0,05 & $0,22 * *$ \\
\hline Fator Geral do CPT & $0,20 * *$ & $0,24^{* *}$ & 0,12 & $0,19 * *$ & $-0,01$ & $0,22 * *$ \\
\hline
\end{tabular}

Notas: $\mathbf{1}$ = Interativa; $\mathbf{2}$ = Normativa; $\mathbf{3}$ = Suprapessoal; $\mathbf{4}$ = Existência; $\mathbf{5}$ = Experimentação; $\mathbf{6}$ = Realização. ${ }^{*}$ Correlação estatisticamente significativa a um $\mathrm{p}<0,05 ; * *$ Correlação estatisticamente significativa a um $\mathrm{p}<0,01$.

Completando as análises acerca do padrão de associação entre as dimensões do CPT e os valores humanos, realizaram-se regressões múltiplas (método enter). Foi introduzido como variável critério cada fator do PTGI, e como variáveis preditoras as subfunções valorativas que apresentaram correlação significativa com as dimensões analisadas. $\mathrm{Na}$ Tabela 2 são mostrados os resultados.

Tabela 2 - Preditores das dimensões do CPT

\begin{tabular}{|c|c|c|c|c|c|c|}
\hline \multicolumn{7}{|l|}{ Fatores PTGI } \\
\hline & Preditores & $\mathbf{R}^{2}$ ajustado & $\mathbf{F}$ & B & B & $T$ \\
\hline & \multicolumn{6}{|c|}{ mudança } \\
\hline \multirow{4}{*}{ Relação com os outros } & Interativa & 0,04 & $8,81^{*}$ & 0,67 & 0,20 & $2,97 * *$ \\
\hline & Normativa & 0,05 & $12,55^{* *}$ & 0,62 & 0,24 & $3,54 * *$ \\
\hline & Existência & 0,02 & $5,53 *$ & 0,61 & 0,16 & $2,35^{*}$ \\
\hline & Realização & 0,02 & $6,01 * *$ & 0,45 & 0,17 & $2,45^{* *}$ \\
\hline \multirow{3}{*}{ Novas Possibilidades } & Suprapessoal & 0,02 & $4,21 *$ & 0,36 & 0,14 & $2,05^{*}$ \\
\hline & Existência & 0,01 & $3,97 *$ & 0,35 & 0,14 & $1,99 *$ \\
\hline & Realização & 0,02 & $6,03 * *$ & 0,31 & 0,17 & $2,46 * *$ \\
\hline \multirow[b]{2}{*}{ Mudança Pessoal } & Existência & 0,02 & $4,19 *$ & 0,26 & 0,14 & $2,04^{*}$ \\
\hline & Realização & 0,04 & $9,91 *$ & 0,28 & 0,22 & $3,15^{*}$ \\
\hline \multirow[b]{2}{*}{ Mudança Espiritual } & Normativa & 0,16 & $39,58 * *$ & 0,37 & 0,40 & $6,29 * *$ \\
\hline & Existência & 0,02 & $4,40 *$ & 0,19 & 0,16 & $2,10 *$ \\
\hline \multirow{3}{*}{ Apreciação da Vida } & Interativa & 0,04 & $8,97^{*}$ & 0,27 & 0,21 & $2,99 *$ \\
\hline & Existência & 0,02 & $4,52 *$ & 0,22 & 0,15 & $2,13^{*}$ \\
\hline & Realização & 0,04 & $10,15^{*}$ & 0,23 & 0,22 & $3,19 *$ \\
\hline \multirow{4}{*}{ Fator Geral do CPT } & Interativa & 0,04 & $8,59 *$ & 1,52 & 0,20 & $2,98^{*}$ \\
\hline & Normativa & 0,06 & $12,94 * *$ & 1,41 & 0,24 & $3,60 * *$ \\
\hline & Existência & 0,03 & $7,93 *$ & 1,63 & 0,19 & $2,82^{*}$ \\
\hline & Realização & 0,04 & $9,90 *$ & 1,30 & 0,22 & $3,15^{*}$ \\
\hline
\end{tabular}

Notas: ${ }^{*} p<0,05 ; * *<0,001$ 
De acordo com a Tabela 2, pode-se constar que as subfunções interativa, normativa, existência e realização, explicaram o fator relação com os outros da escala do PTGI; as subfunções suprapessoal, existência e realização explicaram o fator novas possibilidades; as subfunções existência e realização explicaram o fator mudança pessoal; as subfunções normativa e existência explicaram o fator mudança espiritual e por sua vez o fator apreciação da vida foi explicado pelas subfunções interativa, existência e realização . Finalmente, levando em consideração o fator geral do CPT, as subfunções interativa $(\theta=$ $0,20 ; p<0,05)$, normativa $(\theta=0,24 ; p<0,001)$, existência $(\theta=0,19 ; p<0,05)$ e realização $(\theta$ $=0,22 ; p<0,05)$ o explicaram.

\section{Discussão}

A subfunção existência, representada pelos valores: saúde, sobrevivência e estabilidade pessoal, demostrou poder explicativo em relação a todas as cinco dimensões do CPT. Este resultado pode ser justificado, pois segundo Gouveia $(2003,2013)$ os indivíduos que a priorizam buscam como propósito principal o de assegurar as condições básicas para a sobrevivência biológica e psicológica. Neste processo, as crenças que foram desestruturadas pela vivência do evento adverso passam a ser reexaminadas e os esquemas cognitivos reestruturados, facilitando, assim, o desenvolvimento do fenômeno CPT.

O índice geral do CPT, quanto ao tipo motivador idealista, foi predito, apenas, pela subfunção interativa. Esta relação fundamenta-se tendo em vista que pessoas que primam por tal subfunção, sobretudo pelo valor apoio social, tem maior facilidade com o processo de enfrentamento e adaptação bem sucedida de experiências traumáticas (Ramos \& Leal, 2013). Pois, a busca pelo suporte social providenciará a oportunidade do indivíduo contar repetidamente a sua história, oferecendo novas perspectivas sobre a mesma, possibilitando a atribuição de significado e a criação de uma nova e integradora narrativa de vida (Duarte, 2012).

Todas as subfunções do tipo motivador materialista explicam o fator geral do CPT. Esta relação é teoricamente coesa, pois, estes valores são mais priorizados por pessoas que apresentam uma orientação voltada para ideias práticas e metas específicas. Logo, aquelas que passaram por uma situação adversa, tendem a priorizar a sua sobrevivência e sua existência. Além de ser característica de regiões menos privilegiadas (Gouveia, 2013; Medeiros, 2011; Soares, 2013), situação das cidades do nordeste brasileiro, onde foi feita a coleta de dados.

As relações encontradas apresentaram magnitudes consideradas fracas, porém satisfatórias, tendo em vista a natureza do construto, além de serem raras as correlações acima de 0,30 na Psicologia (Gouveia, Santos, \& Milfont, 2009). Em síntese, pode-se considerar, portanto, que os resultados da presente pesquisa foram, em geral, congruentes com a literatura especializada.

Contudo, como em todo estudo cientifico, neste encontram-se limitações. Um exemplo disto é a amostra por conveniência ou acidental (Cozby, 2003), não devendo assim generalizar os resultados. Outra limitação relaciona-se com a incerteza da magnitude do sofrimento do acontecimento adverso apontado pelos indivíduos que compõe a amostra, não sendo possível compor uma amostra só com pessoas que enfrentaram situações que colocaram a vida em risco, contudo, tendo em vista o caráter subjetivo do trauma (Madeira, 
2013) esta pesquisa aborda situações cotidianas e relevantes para o trabalho da Psicologia. Promovendo o enfrentamento da situação e ressignificação do evento, buscando novos rumos e uma melhor adaptação (Tedeschi \& Calhoun, 2004).

Os resultados obtidos nesta investigação não devem ser encarados como um ancoradouro, mas antes, como uma contribuição para um conhecimento preliminar da ocorrência de CPT, ressalta-se a importância deste estudo. Sobretudo, por poder contribuir com a produção científica que envolve a temática, a qual ainda, mesmo sendo crescente, é passiva de incongruências (Lindstrom, Cann, Calhoun \& Tedeschi, 2013).

Considerando a importância dos valores humanos no processo comportamental e a relevante relação com o CPT, enfatiza-se a relevância em trabalhos preventivos e educação terapêutica. Sob à luz da Psicologia Positiva, favorece o trabalho clínico do psicólogo, demonstrando possibilidades de intervenções potencializadoras de forças e virtudes, favorecendo uma adaptação saudável para as pessoas que vivenciaram uma situação traumática, porém reconhece suas forças, obtêm novas aprendizagens e oportunidades. Tornando-se mais fortes e preparadas para vivências incontroláveis, potencialmente traumática, ao longo da vida (Calhoun \& Tedeschi, 2012).

Esta pesquisa revela a relevância do tema, especialmente na busca da compreensão de antecedentes das aprendizagens obtidas pelas pessoas nas situações de sofrimento, as quais favoreceram de alguma forma o crescimento pessoal. Dessa forma, contribui com evidências cientificas para o equilíbrio do foco dado aos estudos na Psicologia, apresentando um novo olhar, para além do desenvolvimento de patologias, com possibilidades de mudanças positivas como resultado da luta frente ao trauma (Fonseca, 2011). Podendo ser favorecido pela promoção e mudanças das prioridades valorativas, dada a sua importância para a compreensão das ações, julgamentos, escolhas e opiniões das pessoas (Gouveia, 2013; Rokeach, 1973). Por fim, os valores apresentaram-se como uma relevante variável explicadora do CPT, demonstrando, assim, a sua centralidade nos estudos da Psicologia Social.

\section{Conclusões}

Os achados desta investigação diversificam os estudos feitos com o CPT. Os valores humanos, os quais tendo como característica uma maleabilidade quanto as suas prioridades ao longo da vida (Gouveia, Vione, Milfont, \& Fischer, 2015) devem ser encarados como uma importante ferramenta no trabalho clínico, em intervenções terapêuticas de prevenção e promoção de saúde. Principalmente tratando-se de uma psicoeducação que objetive proporcionar mudanças nas prioridades valorativas, direcionando para as que condizem com um melhor enfrentamento da vivência do trauma. Logo, resultados de estudos, a exemplo deste, devem ser encarados como referência nesta contribuição.

Enfatizando o poder preditivo dos valores humanos, os quais tem demonstrado serem um importante construto no estudo da Psicologia Social, sobretudo na explicação de diversas variáveis psicossociais. Por fim, os resultados obtidos também corroboram com o que a Psicologia Positiva se destina à uma mudança de foco, atribuindo importância para as respostas positivas frente ao trauma, com a finalidade de desenvolver potencialidades e virtudes humanas, facilitando que as pessoas vivam mais plena e satisfatoriamente suas vidas. 


\section{Referências}

Bert, S., C. (2011). The Influence of Religiosity and Spirituality on Adolescent Mothers and Their Teenage Children. Journal Youth Adolescence 40, 72-84. Recuperado de http://link.springer.com/article/10.1007\%2Fs10964-010-9506-9.

Calhoun, L. G., \& Tedeschi, R. G. (2012). Crescimento Pós-traumático em intervenções clínicas cognitivocomportamentais. In V. E. Caballo (Ed.). Manual para tratamento cognitivo-comportamental dos transtornos psicológicos da atualidade: Intervenções em crise, transtornos da personalidade e do relacionamento e psicologia da saúde. (pp. 29-47). São Paulo: Santos.

Calhoun, L. G., Tedeschi, R. G., Cann, A., \& Hanks, E. (2010). Positive outcomes following bereavement: Paths to posttraumatic growth. Psychologica Belgica, 50(1\&2), 125-143. Recuperado de http://ptgi.uncc.edu/files/2015/01/Positive-outcomes-following-bereavement.pdf

Cann, A., Calhoun, L. G., Tedeschi, R. G., Taku, K., Vishnevsky, T., Triplett, K. N., \& Danhauer, S. C. (2010). A short form of the Posttraumatic Growth Inventory. Anxiety, Stress, and Coping, 23, 127-137. Recuperado de: http://www.tandfonline.com/doi/pdf/10.1080/10615800903094273?redirect=1.

Cozby, P. C. (2003). Métodos de pesquisa em ciências do comportamento. São Paulo: Atlas.

Duarte, A. M. P. (2012). Experiência do Luto e Crescimento Pós-Traumático à luz da perspectiva da Vinculação numa amostra de estudantes universitários. Dissertação de Mestrado, Centro Regional de Braga, Faculdade de Filosofia, Universidade Católica Portuguesa, Braga, Portugal.

Fonseca, C. C. (2011). Crescimento Pós-Traumático: O impacto de falar sobre os aspectos positivos de uma experiência traumática. Dissertação de mestrado. Faculdade de psicologia, Universidade de Lisboa, Lisboa, Portugal.

Fredrickson, B. L. (2004). Gratitude, like other positive emotions, broadens and builds. In R. A. Emmons \& M. E. McCullough (Eds.) The psychology of gratitude (pp. 145-166). New York: Oxford University Press. R.

Gall, T. L., Kristjansson, E., Charbonneau, C, \& Florack, P. (2009). A longitudinal study on the role of spirituality in response to the diagnosis and treatment of breast cancer. Journal of Behavioral Medicine, 32, 174186. Recuperado de http://link.springer.com/article/10.1007\%2Fs10865-008-9182-3.

Gouveia, V. V. (1998). La naturaleza de los valores descriptores del individualismo e del colectivismo: Una comparación intra e intercultural. Tese de Doutorado. Departamento de Psicologia Social, Universidade Complutense de Madri, Espanha.

Gouveia, V. V. (2003). A natureza motivacional dos valores humanos: Evidências acerca de uma nova tipologia. Estudos de Psicologia, 8, 431-443. Recuperado de http://www.scielo.br/pdf/epsic/v8n3/19965.pdf.

Gouveia, V.V. (2013). Teoria funcionalista dos valores humanos: Fundamentos, aplicações e perspectivas. São Paulo: Casa do Psicólogo.

Gouveia, V. V., Milfont, T. L., Fischer, R., \& Santos, W. (2008). Teoria funcionalista dos valores humanos. In M. L. M. Teixeira (Org.). Valores humanos e gestão: novas perspectivas (pp. 47-80). São Paulo: Senac.

Gouveia, V. V., Santos, W. S., \& Milfont, T. L. (2009). O uso da estatística na avaliação psicológica: Comentários e considerações práticas. In C. S. Hutz (Ed.). Avanços e polêmicas em avaliação psicológica: Em homenagem a Jurema Alcides Cunha (pp. 127-155). São Paulo, SP: Casa do Psicólogo.

Gouveia, V. V., Vione, K. C., Milfont, T. L., \& Fischer, R. (2015). Patterns of value change during the life span: Some evidence from a functional approach to values. Personality and Social Psychology Bulletin, $1-15$. Recuperado de http://psp.sagepub.com/content/early/2015/07/17/0146167215594189.full.pdf+html.

Inglehart, R. (1991). El cambio cultural en las sociedades industriales avanzadas. Madri, España: Siglo XXI.

Lau, J. T. F., Yeung, N. C. Y., Yu, X., Zhang, J., Mak, W. W. S., Lui, W. W. S. et al. (2013). Psychometric properties of the Chinese version of the Revised Posttraumatic Growth Inventory for Children (PTGI-C-R). AsiaPacific Journal of Public Health, 27(2), 310-320. Recuperado de http://aph.sagepub.com/content/early/2013/03/14/1010539513479967.abstract. 
Lindstrom, C. M., Cann, A., Calhoun, L. G., \& Tedeschi, R. G. (2013). The relationship of core belief challenge, rumination, disclosure, and sociocultural elements to posttraumatic growth. Psychological Trauma, 5(1), 50-55. Recuperado de http://psycnet.apa.org/index.cfm?fa=buy.optionToBuy\&id=2011-06103-00.

Madeira, S. R. M. (2013). Relação entre as características da situação vivida, suporte social e resiliência, após a vivência de um acontecimento potencialmente traumático. Dissertação de mestrado. Universidade de Lisboa, Faculdade de Psicologia.

Maia, A., \& Resende, C. (2008). Dados de exposição potencialmente traumática na população portuguesa utilizando as versões portuguesas do Life Events Checklist e o Life Stressor Checklist - Revised. In Avaliação Psicológica: Formas e contextos: Actas da XIII Conferência Internacional de Avaliação Psicológica. Braga: Psiquilibrios Edições.

Medeiros, E. D. (2011) Teoria funcionalista dos valores humanos: Testando sua adequação intra e interculturalmente. Tese de Doutorado. Departamento de Psicologia, Universidade Federal da Paraíba, João Pessoa, PB.

Medeiros, E. D., Couto, R. N., Fonseca, P. N., Silva, P. G. N., \& Medeiros, P. B. C. (No prelo). Posttraumatic Growth Inventory (PTGI): Adaptação e validade fatorial no nordeste brasileiro. Psico-USF.

Osei-Bonsu, P. E., Weaver, T. L., Eisen, S. V., \& Wal, J. S. V. (2012). Posttraumatic Growth Inventory: Factor structure in the context of DSM-IV traumatic events. International Scholarly Research Network, 2012 (2012). Recuperado de http://downloads.hindawi.com/journals/isrn.psychiatry/2012/937582.pdf.

Ramos, C., \& Leal, I., (2013). Posttraumatic growth in the aftermath of trauma: A literature review about related factors and application contexts. Psychology, Community \& Health, 2(1), 43-54. Recuperado de http://pch.psychopen.eu/article/view/39/pdf.

Reppold, C. T., Gurgel, L. L., \& Schiavon, C., C. (2015). Research in Positive Psychology: a Systematic Literature Review. Psico-USF, Bragança Paulista, 20(2) 275-285. Recuperado de http://pch.psychopen.eu/article/view/39/35.

Rokeach, M. (1973). The nature of human values. New York: Free Press.

Ros, M. (2006). Psicologia social dos valores humanos: uma perspectiva histórica. In M. Ros \& V. V. Gouveia (Cords.). Psicologia social dos valores humanos: Desenvolvimentos teóricos, metodológicos e aplicados (pp. 23-53). São Paulo: Editora Senac, São Paulo.

Schwartz, S. H. (1992). Universal in the content and structure of values: Theoritical advances and empirical tests in 20 countries. In M. P. Zanna (Org.). Advanced in Experimental Social Psychology (pp. 1-65). Nova York: Academic Press.

Schwartz, S. H. (2012). An overview of the Schwartz theory of basic values. Online Readings in Psychology and Culture, 2, 1-20. Recuperado de http://dx.doi.org/10.9707/2307-0919.1116.

Subandi, M. A., Achmad, T., Kurniati, T., \& Febri, R. (2014). Spirituality, gratitude, hope and post-traumatic growth among the survivors of the 2010 eruption of Mount Merapi in Java, Indonesia. Australasian Journal of Disaster and Trauma Studies 18(1), 19-26. Recuperado de http://www.massey.ac.nz/ trauma/issues/2014-1/AJDTS_18-1_Subandi.pdf.

Soares, A. K. S. (2013). Valores humanos e bullying: Um estudo pautado na congruência entre pais e filhos. Dissertação de Mestrado não publicada. Departamento de Psicologia, Universidade Federal da Paraíba, João Pessoa, PB.

Tabachnick, B., \& Fidell, L. S. (2012). Using multivariate statistics (6 ed.). Boston: Pearson.

Taku, K., Kilmer, R. P., Cann, A., Tedeschi, R. G., \& Calhoun, L. G. (2012). Exploring Posttraumatic Growth in Japanese Youth. Psychological Trauma: Theory, Research, Practice, and Policy, 4(4), 411-419. Recuperado de http://psycnet.apa.org/journals/tra/4/4/411.

Tedeschi, R. G. (2011). Posttraumatic Growth in Combat Veterans. Journal of Clinical Psychology in Medical Settings, 18, 137-144.

Tedeschi, R. G., \& Calhoun, L. G. (1996). The Posttraumatic Growth Inventory: Measuring the positive legacy of Trauma. Journal of Traumatic Stress, 9(3), 455-471. Recuperado de http://www.ncbi.nlm.nih.gov/pubmed/8827649. 
Tedeschi, R. G., \& Calhoun, L. G. (2004). Posttraumatic Growth: Conceptual Foundations and Empirical Evidence. Psychological Inquiry, 15, 1-18. Recuperado de https://ptgi.uncc.edu/files/2013/01/PTGConceptual-Foundtns.pdf.

Thege, B., K., Kovács, E., \& Balog, P. (2013). A bifactor model of the Posttraumatic Growth Inventory. Health Psychology \& Behavioral Medicine, 1, 529-540. Recuperado de http://www.tandfonline.com/doi/abs/10.1080/21642850.2014.905208.

Triplett, K., Tedeschi, R., Cann, A., Calhoun, L. \& Reeve, C. (2012). Posttraumatic growth, meaning in life, and life satisfaction in response to trauma. Psychological Trauma: Theory, Research, Practice, and Policy, 4(4), 400-410. Recuperado de http://psycnet.apa.org/journals/tra/4/4/400/.

Vázquez, C., Castilla, C., \& Hervás, G. (2008). Reacciones frente el trauma: vulnerabilidad, resistencia y crecimiento. In E. Fernández-Abascal (Ed.). Las Emociones Positivas (pp. 375-392). Madrid: Pirámide.

Vázquez, C. (2013). Positive Psychology and its enemies: A reply based on scientific evidence. Papeles del Psicólogo, 34, 3-25. Recuperado de

https://www.researchgate.net/publication/259257461_Positive_psychology_and_its_enemies_A_reply _based_on_scientific_evidence.

Wolfe, J., Kimerling, R. Brown, P. Chresman K., \& Levin, K. (1996). Psychometric review of the life stressor checklist-revised. In B. Stamm (Ed.). Instrumentation in stress, trauma, and adaptation, 144-151. Sidran Press, Lutherville, MD.

Wu, X., Zhou, X., Liu, L., \& Chen, J. (2014). Social support and deliberate rumination mediate the relationship between gratitude and post-traumatic growth in adolescents at 4.5 years after Wenchuan earthquake. Psychological Science, 37, 1148-1153. Recuperado de http://www.psysci.org/EN/article/showArticleFile.do?attachType=HTML\&id=9319.

Zanon, C., Borsa, J. C., Bandeira' D. R., Hutz, C. S. (2012). Relações entre pensamento ruminativo e facetas do neuroticismo. Estudos de Psicologia (Campinas), 29(2). Recuperado de http://www.scielo.br/pdf/estpsi/v29n2/a03v29n2.pdf.

Zhou, X., \& Wu, X. (2015). Longitudinal relationships between gratitude, deliberate rumination, and posttraumatic growth in adolescents following the Wenchuan earthquake in China. Scandinavian Journal of Psychology, 56, 567-572. Recuperado de http://onlinelibrary.wiley.com/doi/10.1111/sjop.12237/pdf. 